\title{
ARTICLE
}

\section{Thymopentin-loaded phospholipid-based phase separation gel with long-lasting immunomodulatory effects: in vitro and in vivo studies}

\author{
Ting Zhang ${ }^{1,2}$, Xian-yan Qin ${ }^{1}$, Xi Cao ${ }^{1}$, Wen-hao $\mathrm{Li}^{1}$, Tao Gong ${ }^{1}$ and Zhi-rong Zhang ${ }^{1}$
}

\begin{abstract}
Thymopentin (TP5) is an effective immunomodulatory agent for autoimmune disease that has been used clinically for decades. However, its application is greatly limited by its extremely short half-life in vivo, poor membrane permeability and extensive metabolism in gastrointestinal tract, resulting in repeated injection and poor patient compliance. In the present study, we developed a TP5-loaded, phospholipid-based phase separation gel (PPSG) to achieve sustained drug release profile and longlasting therapeutic effects. We firstly demonstrated the physiochemical characteristics of PPSG before and after phase transition by examining the viscosity and morphology change caused by the phase transition. Moreover, the PPSG exerted a low cytotoxicity in L929 cells and HUVECs, suggesting the biocompatibility of PPSG. A month-long drug release profile of TP5 PPSG was observed both in vitro and in vivo, revealing its sustained and controlled drug release property. Most importantly, in cyclophosphamide-induced immunosuppressive rats, a single dose of TP5 PPSG $(15 \mathrm{mg} / \mathrm{kg}, \mathrm{sc})$ injected could normalize their T-SOD levels and CD4+/CD8+ ratio; such an immunoregulatory effect was comparable to that produced by repeated injection of TP5 solution $(0.6 \mathrm{mg} / \mathrm{kg}$ per day, sc) for 14 consecutive days. Thus, TP5 PPSG has a great potential for sustained delivery of TP5 in clinical use because of its simple manufacture process, good biocompatibility and long-lasting immunomodulatory efficacy, which could greatly improve patient compliance.
\end{abstract}

Keywords: thymopentin; phospholipid-based phase separation gel (PPSG); phase transition; sustained drug release; pharmacokinetics; immunoregulatory effect

Acta Pharmacologica Sinica (2019) 40:514-521; https://doi.org/10.1038/s41401-018-0085-8

\section{INTRODUCTION}

Thymopentin (TP5) is a synthetic pentapeptide (Arg-Lys-AspValTyr, Mw $=679.77$ ) corresponding to the sequence of thymic hormone thymopoietin (residues 32-36) [1]. TP5 showed similar immunomodulatory bioactivity to thymopoietin for treatment of autoimmune diseases, including chronic lymphocytic leukemia [2], rheumatoid arthritis [3], cancer immunodeficiency [4], acquired immunodeficiency syndrome (AIDS) [5], and chronic heart failure [6]. The mechanism that has been demonstrated is that TP5 can promote the differentiation of thymocytes and affect the function of mature T cells [7-9]. However, TP5 has a very short half-life in vivo (less than $30 \mathrm{~s}$ ), poor membrane permeability and extensive metabolism in the gastrointestinal tract [10]. Therefore, it is usually marketed as a freeze-dried powder for intramuscular or hypodermic injection. Autoimmune diseases are generally chronic and long-lasting, so patients would suffer the pains of repeated injections for one to six months [11], which has greatly reduced patient compliance and restricted the clinical application of TP5. Moreover, it has been reported that the potency of TP5 in vivo can be increased by slow infusion [12].

Various novel drug delivery systems have been developed for TP5 in previous studies. For example, orally administered nanoparticles [13, 14] and inhalable microparticles [11] have been prepared to avoid the pain of injection. Polymer nanoparticles [15], implants [16], and hydrogels [17] have been prepared for sustained TP5 delivery to reduce the injection frequency. However, some problems still exist. Because of hydrophilicity, TP5-loaded nanoparticles and hydrogels often showed a low entrapment efficiency and a high burst release profile. Microparticles often require a complex preparation process and a high cost. Moreover, the materials for novel drug vehicles are usually non-commercial, expensive, or non-biodegradable. For example, $\mathrm{Wu}$ et al. prepared polymer nanoparticles with a high loading efficiency and improved pharmacokinetics and pharmacodynamics properties. Nevertheless, the manufacturing operation was complex, and the main material, PHBHHX, was noncommercial, which greatly limited its clinical usage [15]. Recently, Lin et al. developed a TP5-loaded dissolving microneedle array to provide safe transdermal delivery. However, the preparation of microneedle arrays needs a specialized model, and repeated daily administration was also required [18]. Most importantly, a toxic organic solution (e.g., chloroform and methylene chloride) is often used in the manufacturing process and could not be completely removed, which is a potential threat to a patient's health [16].

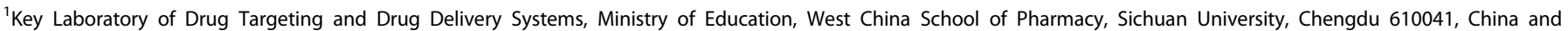
${ }^{2}$ Department of Pharmacy, West China Hospital, Sichuan University, Chengdu 610041, China

Correspondence: Tao. Gong (gongtaoy@126.com)
}

Received: 1 May 2018 Accepted: 20 June 2018

Published online: 12 July 2018 
Therefore, a novel TP5-loaded sustained drug delivery system that could be easily prepared with biodegradable, safe, and lowcost materials is necessary. Phospholipid gels have been previously studied as a sustained drug delivery system because of the availability, non-toxicity and biocompatibility of phospholipids. For example, Zhong et al. [19] prepared a vesicular phospholipid gel for TP5 delivery. However, this system contained excessive water and a small amount of phospholipid to produce a low viscosity for injection, so its burst release could be relatively high and its pharmaceutical effect was of relatively short duration (i.e., a week). Recently, our group has developed a novel phospholipid-based phase separation gel (PPSG) that was a solvent-induced in situ forming gel and achieved a good longterm drug delivery property [20]. This gel formulation is based on high-content phospholipid, little anhydrous ethanol, and injection-grade oil, and it could be prepared easily by a onestep stirring method. PPSG is in solution initially, and upon exposure to an aqueous environment, it transforms into a gel, which serves as a drug depot for the long-term release of a drug after injection with a hypodermic.

In the present study, for the first time, a TP5-loaded PPSG was employed for sustained drug delivery and a long-lasting immunoregulatory effect and was studied systematically both in vitro and in vivo. The characteristics of PPSG before and after a phase transition were first studied using viscosity determination, atomic force microscopy (AFM), and differential scanning calorimetry (DSC). The cellular toxicity of PPSG was also investigated in the L929 and HUVEC cell lines. Moreover, the drug release behavior of TP5-loaded PPSG was studied both in vitro and in vivo. Finally, a pharmacodynamics study was carried out on a cyclophosphamide-induced immunodepression rat model to determine the long-term immunoregulatory effect of TP5-PPSG.

\section{MATERIALS AND METHODS}

Materials

TP5 and 5-FAM-TP5 were kindly given as gifts by the Chengdu Kaijie Biotechnologies Co. Ltd. (Chengdu, China). Egg yolk lecithin (E80) was provided by Lipoid Co. Ltd (Ludwigshafen, Germany), and medium chain triglycerides (MCT) were purchased from Beiya Medical Oil Co. Ltd. (Tieling, China). A total superoxide dismutase (T-SOD) kit was bought from Nanjing Jiancheng Bioengineering Institute (Najing, China). Anti-rat CD3-APC, anti-rat CD8b-PE, and anti-rat CD4-FITC were purchased from BD Biosciences (New Jersey, USA). All other reagents were of analytical grade or better.

Cells and animals

Healthy female Wistar rats $(180-220 \mathrm{~g})$ were purchased from the Laboratory Animal Centre of Sichuan University (Chengdu, China). All rats were housed in a specific pathogen-free, light-cycled and temperature-controlled facility, and five animals were housed per cage with free access to food and water. The rats were acclimatized for one week prior to use in experiments. All the animal experiments were approved by the Institutional Animal Care and Ethic Committee of Sichuan University.

In addition, mouse fibroblasts (L929) and human umbilical vein endothelial cells (HUVEC) were purchased from the Shanghai Institutes for Biological Sciences (Shanghai, China) and cultured in PRIM-1640 medium (HyClone, USA) containing penicillin (100 lU/ $\mathrm{mL}$ ), streptomycin $(100 \mu \mathrm{g} / \mathrm{mL})$, and $10 \%$ fetal bovine serum (Fu Meng Gene, Shanghai, China) in a humidified atmosphere containing $5 \% \mathrm{CO}_{2}$ at $37^{\circ} \mathrm{C}$. After being counted and passaged, cells in logarithmic phase were used in this study.

Preparation of PPSG and TP5-PPSG

PPSG was prepared by a simple one-step stirring method according to our previous study[20]. In brief, E80, absolute ethanol and $\mathrm{MCT}$ in a weight ratio of 70:15:15 were mixed and agitated vigorously for two hours at room temperature. Water bath sonication was used to remove air bubbles when necessary, resulting in a homogeneous PPSG. In addition, when loading the TP5 into PPSG, ethanol was replaced by an acetate buffer ( $\mathrm{pH} 6.0)$ ethanol $(15 / 85, v / v)$ mixture to dissolve the TP5 and keep it stable, and the TP5 concentration in PPSG was $5 \mathrm{mg} / \mathrm{mL}$.

Meanwhile, according to our preliminary in vivo study (data not shown), the PPSG contained 51.1\% E80, 48.7\% PBS, 0.1\% MCT and $0.1 \%$ ethanol after a steady phase transition. Based on this composition, the phase-transformed PPSG (gelated PPSG) was formulated by mixing these components.

\section{Characterization of PPS}

Viscosity. The viscosity of PPSG after the phase transition was determined at room temperature with a method described in a previous study (DV-C, Brookfield Engineering Laboratories, Inc., USA) [20].

Atomic force microscopy (AFM). The morphology of PPSG before and after the phase transition was observed by AFM. The phase transition process was induced in rats. First, $1 \mathrm{~mL}$ of PPSG was injected subcutaneously into a rat and withdrawn after four hours, and then, a frozen section (thickness, $50 \mu \mathrm{m}$ ) was prepared. The section was adhered to a freshly exfoliated mica sheet. After natural air drying overnight, the morphology was examined via AFM (Model SPM9600, Shimadzu, Japan) using the phase imaging mode [21].

Differential scanning calorimetry (DSC). All of the samples, including E80, MCT, $\mathrm{H}_{2} \mathrm{O}$, ethanol, PPSG- 70-(+), and gelated PPSG, were placed into a hermetically sealed platinum crucible for DSC, and thermograms were recorded from $0-300^{\circ} \mathrm{C}$ at a heating rate of $10^{\circ} \mathrm{C} / \mathrm{min}$ under a constant protective nitrogen atmosphere $(0.2 \mathrm{~mL} / \mathrm{min})$. This study employed a differential scanning calorimeter (Mettler Toledo, USA), and $10 \mathrm{mg}$ was needed for each sample.

Cytotoxicity assay of PPSG

A cytotoxicity assay for PPSG was conducted in L929 cells and HUVECs using an extraction colony assay, which is usually used for the toxicity evaluation of implants [22, 23]. Briefly, $1 \mathrm{~mL}$ of PPSG and $6 \mathrm{~mL}$ of PRIM-1640 culture medium were mixed and then incubated at $37^{\circ} \mathrm{C}$ for $24 \mathrm{~h}$, following which the tube was centrifuged and the supernatant containing the extracted PPSG was retained for further study. After dilution with culture medium and filtration through a $0.22 \mu \mathrm{m}$ millipore filter, different concentrations of PPSG extract $(25,50,75$, and 100\%) were obtained.

L929 cells or HUVECs in logarithmic phase were seeded in 96well plates at a density of $1 \times 10^{4}$ cells/well/0.1 mL. After $24 \mathrm{~h}$ of incubation for cell attachment, the cells were exposed to cell culture medium containing various concentrations of PPSG extract $(25,50,75$, and $100 \%)$ for another $24 \mathrm{~h}$ of incubation. Cells untreated with PPSG extract served as the control group. Finally, the cell viability was determined by the MTT assay as previously described [24].

In vitro release study

An in vitro release study was conducted by dynamic dialysis in phosphate-buffered saline containing different concentrations of ethanol $(0,10$, and 20\%) $[25,26]$. Aliquots of $0.2 \mathrm{~g}$ of TP5-PPSG (TP5 concentration $=5.0 \mathrm{mg} / \mathrm{mL}$ ) or $1.0 \mathrm{~mL}$ of TP5 (TP5 concentration $=1.0 \mathrm{mg} / \mathrm{mL}$ ) solution were added to the dialysis bags (molecular weight cut-off $=8-14 \mathrm{kDa}$ ). After the ends were tied, the dialysis bags were immersed in $4 \mathrm{~mL}$ of release medium (PBS or ethanol-containing PBS, $\mathrm{pH} 7.4$ ), followed by shaking on a horizontal shaker $\left(100 \mathrm{rpm}, 37 \pm 0.5^{\circ} \mathrm{C}\right)$. At predetermined time points, the release medium was collected and replaced with $4 \mathrm{~mL}$ 
a

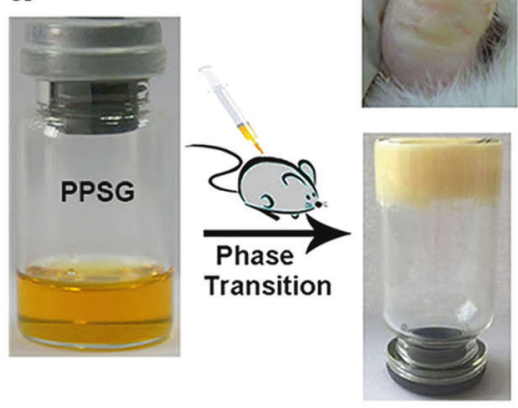

b

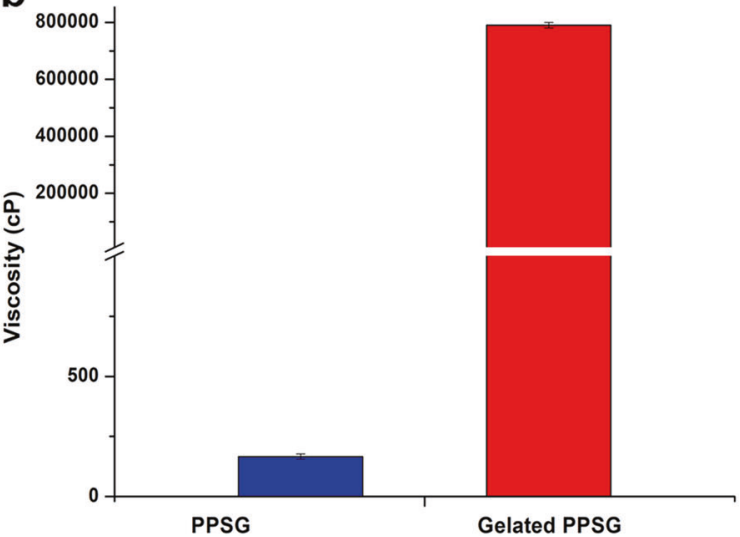

Fig. 1 a Appearance and $\mathbf{b}$ viscosity of PPSG before and after the phase transition

of fresh medium. The collected release medium was diluted to $5 \mathrm{~mL}$ with PBS containing 5\% Triton X-100 and quantified by HPLC (Agilent 1260 infinity, USA). Finally, cumulative release percentage-time curves were drawn to compare the release profile of free TP5 and TP5-PPSG. All measurements were carried out in triplicate.

The HPLC conditions for TP5 detection are described below. A volume of $50 \mu \mathrm{L}$ of each sample was injected into the HPLC system. A Kromasil-C18 reverse phase column $(150 \mathrm{~mm} \times 4.6 \mathrm{~mm}$, $5 \mu \mathrm{m})$ and a mobile phase consisting of phosphate buffer $(0.05 \mathrm{M}$, $\mathrm{pH} 7.0)$ and methanol (86/14, v/v) was used for TP5 separation. The samples were eluted by the mobile phase at a flow rate of $1.0 \mathrm{~mL} / \mathrm{min}$ at $35^{\circ} \mathrm{C}$ and monitored at $276 \mathrm{~nm}$. The peak area was recorded for TP5 quantification using a validated standard curve.

\section{In vivo pharmacokinetics study}

Healthy female Wistar rats were randomly divided into two groups $(n=5)$ and fasted for $12 \mathrm{~h}$ before the subcutaneous injection of a 5-FAM-TP5 solution and 5-FAM-TP5-PPSG at a dosage of $20 \mathrm{mg} / \mathrm{kg}$ body weight. At pre-determined time points, $300 \mu \mathrm{L}$ of blood was collected from the eye socket into heparinized tubes, followed by centrifugation $\left(2500 \times g\right.$ for $\left.5 \mathrm{~min}, 4^{\circ} \mathrm{C}\right)$ to separate the plasma. An aliquot of $100 \mu \mathrm{L}$ of plasma was collected and stored at $-80^{\circ} \mathrm{C}$ for analysis by fluorescence spectrophotometry. The plasma samples were first preprocessed by precipitating the protein with acetonitrile to eliminate interference from the serum and then quantified on a fluorescence spectrophotometer (RF-5301 PC, Shimadzu, Kyoto, Japan) [15], in which the excitation wavelength and emission wavelengths were set to 502 and $539 \mathrm{~nm}$, respectively. To account for the variability of the fluorescence quantification, a regression equation was established in every quantification test, and the methodology was first validated as meeting the quantitative requirements.

In vivo pharmacodynamics study

Healthy male Wistar rats were randomly divided into four groups $(n=5)$, i.e., group 1 was the normal control, group 2 was the immunosuppression control, group 3 was the TP5 solution group and group 4 was the TP5-PPSG group. During the first three consecutive days, rats in groups 2,3 , and 4 were intraperitoneally injected with a cyclophosphamide solution at a dosage of $35 \mathrm{mg} /$ $\mathrm{kg}$ per day to establish the immunosuppression model. During the following two weeks, rats in group 2 were subcutaneously injected with saline once daily, rats in group 3 were given a subcutaneous injection of TP5 solution at a dosage of $0.6 \mathrm{mg} / \mathrm{kg}$ per day, and rats in group 4 were subcutaneously injected with TP5-PPSG at a dosage of $15 \mathrm{mg} / \mathrm{kg}$ on the fourth day only. During the entire test, group 1 was normally fed without drug treatment, and all rats were given free access to food and water. Two weeks after the TP5 injection, the immune status of all of the rats was determined. A volume of $1 \mathrm{~mL}$ of blood sample was taken from the eye socket of each rat into an anticoagulative tube for T-SOD activity and T-lymphocyte subset analysis.

T-SOD activity determination. After sampling, the whole blood was centrifuged $\left(2500 \times g\right.$ for $\left.5 \mathrm{~min}, 4^{\circ} \mathrm{C}\right)$ to separate the plasma, and an aliquot of $20 \mu \mathrm{L}$ of plasma was collected and processed according to the T-SOD kit instructions. Finally, ultraviolet spectrophotometry (CARY100, Varian, Japan) at $550 \mathrm{~nm}$ was used to determine the ultraviolet absorption of the samples, and T-SOD values were calculated for each group.

T-lymphocyte subset analysis. The T-lymphocyte subsets were determined using three-color flow cytometry. In brief, each $100 \mu \mathrm{L}$ anticoagulated blood sample was mixed with $30 \mu \mathrm{L}$ of fluorescent antibodies, which was first diluted 10:1 with PBS (containing $1.25 \mu \mathrm{L}$ of anti-rat CD3-APC, $0.5 \mu \mathrm{L}$ of anti-rat CD4-FITC, and $1.25 \mu \mathrm{L}$ of anti-rat CD8b-PE). After incubation for $15 \mathrm{~min}$ at $70 \mathrm{rpm}$ in the dark, an aliquot of $1 \mathrm{~mL}$ of red blood cell lysis buffer was added to each sample, and the mixture was incubated for another $20 \mathrm{~min}$ in the dark $\left(25^{\circ} \mathrm{C}\right)$. The red blood cells were removed by twice of cell lysis and centrifugation $\left(2000 \times g\right.$ for $\left.5 \mathrm{~min}, 4^{\circ} \mathrm{C}\right)$, after which the cell sediments were washed twice with PBS. Blank blood samples and samples labeled only with anti-rat CD3-APC or anti-rat CD4FITC or CD8b-PE were used as the blank control and fluorescent compensation control, respectively. The cells were resuspended in $0.4 \mathrm{~mL}$ of PBS, followed by a flow cytometry analysis to determine the T-lymphocyte subsets (Cytomics FC 500, Beckman Coulter, USA). Finally, the CD4+/CD8+ ratios were calculated from the amounts of $\mathrm{CD} 3+/ \mathrm{CD} 4+\mathrm{T}$ cell and $\mathrm{CD} 3+/ \mathrm{CD} 8+\mathrm{T}$ cell to determine the therapeutic effect of each group on the immunosuppressed rats.

Data and statistical analysis

The results of all of the tests were expressed as the mean \pm SD (standard deviation). A two-tailed Student's $t$-test was performed for statistical analysis, in which $P<0.05$ indicated that statistical differences existed between two groups, and $P<0.01$ was considered statistically significant different. All tests were conducted in triplicate.

\section{RESULTS AND DISCUSSION}

\section{Characterization of PPSG}

Viscosity. A low viscosity is essential for an injectable gel, while a high viscosity is also important for a phase-transformed gel implant that could serve as a drug depot for sustained drug release. As shown in Fig. 1a, PPSG was transformed from a 
a

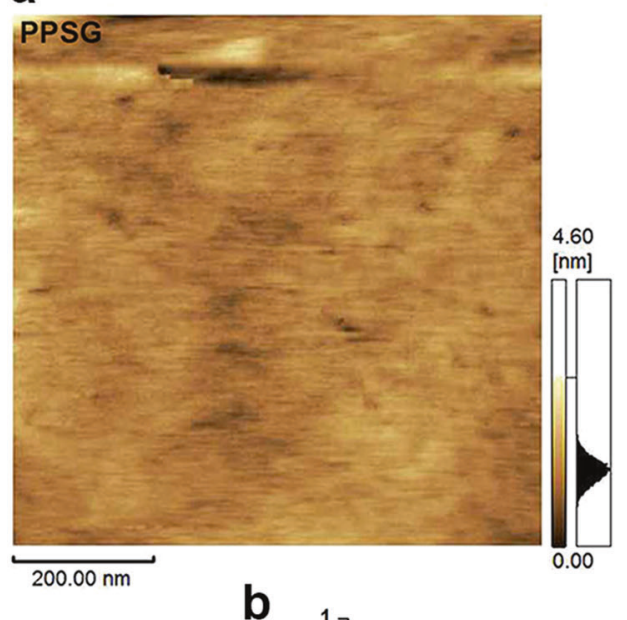

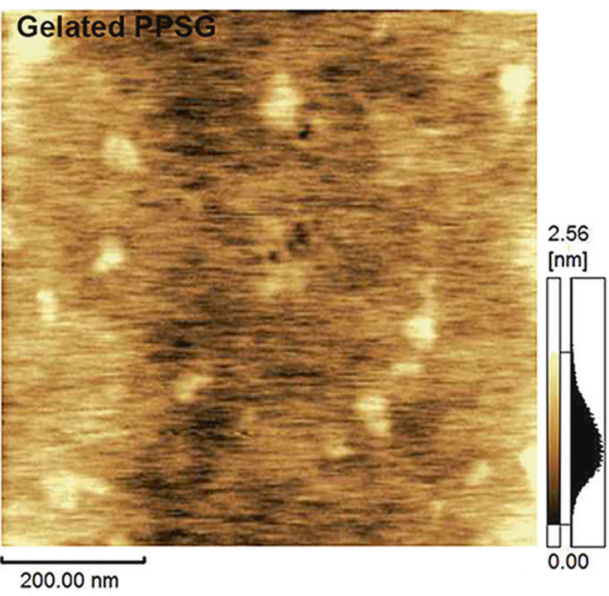

$200.00 \mathrm{~nm}$

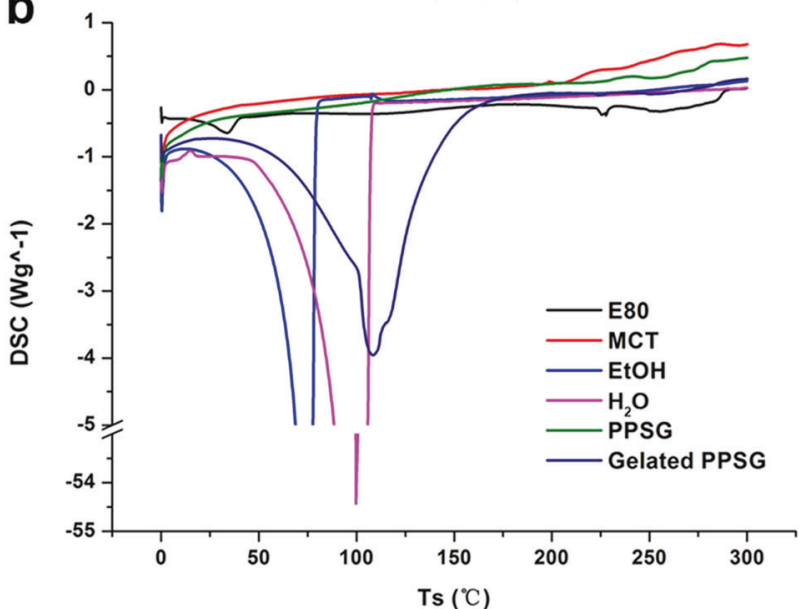

Fig. 2 a Atomic force microscopy (AFM) and b differential scanning calorimetry (DSC) determination of PPSG before and after the phase transition

transparent solution into a gel after the occurrence of a phase transition. The viscosity of the gelated PPSG was determined as $790467 \mathrm{CP}$, which was significantly higher than that of the initial solution state $(165 \mathrm{cP})$ (Fig. 1b). Consequently, the gel implants could act as a barrier for the controlled release of a loaded drug.

AFM. AFM was conducted to study the morphology of PPSG. Fig. 2a shows a homogeneous system image for PPSG, indicating that the phospholipid in PPSG was completely dissolved. Conversely, after the phase transformation, an image of solidified PPSG clearly showed phospholipid sediment particles, which could serve as a depot for sustained drug release after the PPSG undergoes the phase transformation upon exposure to water.

Differential scanning calorimetry. DSC curves (Fig. 2b) showed an endothermic peak at $33.89^{\circ} \mathrm{C}$ for phospholipid E80, which could be its melting point. The $\mathrm{H}_{2} \mathrm{O}$ and ethanol DSC curves showed endothermic peaks at $99.76{ }^{\circ} \mathrm{C}$ and $76.37{ }^{\circ} \mathrm{C}$ that could be the boiling points of $\mathrm{H}_{2} \mathrm{O}$ and ethanol, respectively. The DSC curve of MCT was smooth and had no peaks, indicating that no phase transformation occurred in this process. Similarly, PPSG showed a smooth curve similar to MCT, demonstrating that this system was stable as the temperature increased. In contrast, a wide and blunt endothermic peak at $108.66^{\circ} \mathrm{C}$ appeared in the DSC curve of solidified PPSG, which could have been caused by the boiling of $\mathrm{H}_{2} \mathrm{O}$ and the phase transformation of the gel to the liquid crystalline state when the phospholipid non-polar hydrocarbon chain melted and isomerized. It has been reported that the liquid crystalline phase was always hexagonal and anisotropic $[22,27]$.

In a previous study [26], OCT PPSG $\left(C_{\mathrm{OCT}}=5 \mathrm{mg} / \mathrm{mL}\right)$ and blank PPSG showed almost the same viscosity at the same temperature, and OCT PPSG transformed into a gel upon exposure to water. Both OCT (octapeptide, Mw = 1019.24) and TP5 (pentapeptide, $\mathrm{Mw}=679.77$ ) are low molecular weight peptides, and the drug concentrations in PPSG were relatively low $(5 \mathrm{mg} / \mathrm{mL})$. Therefore, it could be inferred that TP5-loaded PPSG would show a similar phase transformation behavior as PPSG in this study.

\section{Cytotoxicity assay of PPSG}

Fig. 3 shows the cytotoxicity caused by PPSG serial dilutions. Both L929 cells and HUVECs showed no significant cellular toxicity, with a cell viability higher than $80 \%$ when the PPSG extract concentration was $25-75 \%$. Moreover, at the highest extract concentration of $100 \%$, the average cell viabilities of L929 cells and HUVECs were $93.59 \%$ and $65.73 \%$, respectively, which could be attributed to the different tolerances of the cell lines, indicating the inhibition of HUVEC proliferation upon exposure to the PPSG extracts in the first $24 \mathrm{~h}$. An extract cytotoxicity assay is generally conducted to determine the toxicity of solid implants in the body. The primary toxicity of the PPSG extracts could be caused by the small amount of ethanol in PPSG. However, upon exposed to body fluid in vivo, the PPSG implant extract could be diluted over time and the possible toxicity could be eliminated, which we demonstrated in a previous study [20]. 
In vitro release study of TP5-PPSG

Fig. 4 shows the release profiles of a TP5 solution and TP5-PPSG. Fig. 4a shows that a large burst release was observed both in PBS and ethanol-containing PBS medium for the TP5 solution, with a cumulative release percentage above $50 \%$ during the first half hour. Subsequently, the release percentage in all groups increased with time and plateaued at approximately the 8th hour, and the drug release was almost complete within $24 \mathrm{~h}$. The release profile could be divided into two phases, that is, the burst release during the first $6 \mathrm{~h}$ with a high cumulative release percentage (approximately $90 \%$ ) and a subsequent sustained drug release phase that resulted in the nearly complete release of the drug. Fig. 4a also indicates a rapid drug release of the TP5 solution regardless of the ethanol concentration.

In contrast, the TP5 release from PPSG was much slower (Fig. 4b) compared with the solution formulation. The cumulative release percentages of TP5-PPSG within $912 \mathrm{~h}(38 \mathrm{~d})$ in $0 \%, 10$ and $20 \%$ ethanol-containing PBS were $52.3 \%, 71.5 \%$ and $75.8 \%$, respectively. As seen with the TP5 solution, the release profile for TP5-PPSG also had two phases. In the initial burst release phase $(0-6 \mathrm{~h})$, the cumulative drug release percentages in $0 \%, 10$ and $20 \%$ ethanol-containing PBS were $15.9 \%, 20.1 \%$ and $30.5 \%$, respectively, which were much slower than that for the

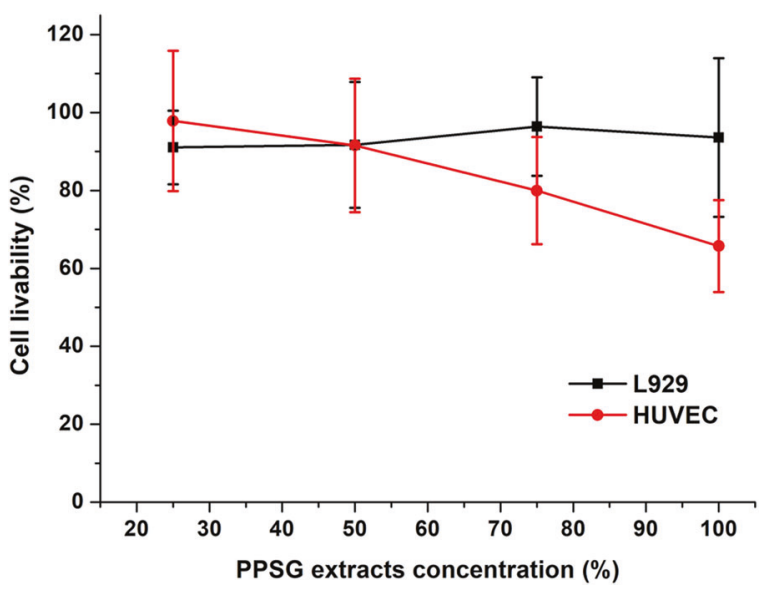

Fig. 3 Cell livability of L929 cells and HUVECs after incubated with various concentrations of PPSG extraction $(25,50,75$, and $100 \%)$ for $24 \mathrm{~h}(n=5)$

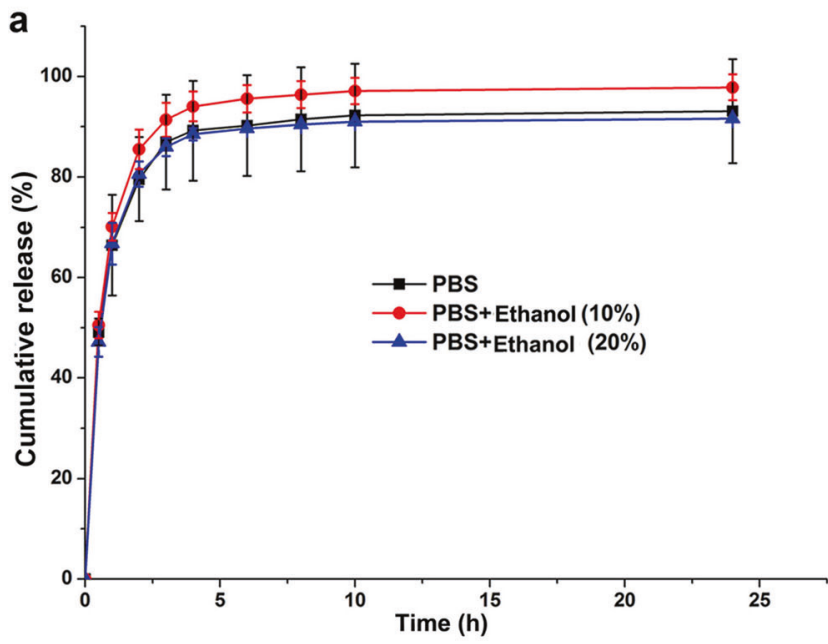

Fig. 4 In vitro release profiles of TP5 solution (a) and TP5-loaded-PPSG (b) in release medium containing different concentrations of ethanol $(0,10$, and $20 \%)(n=3)$

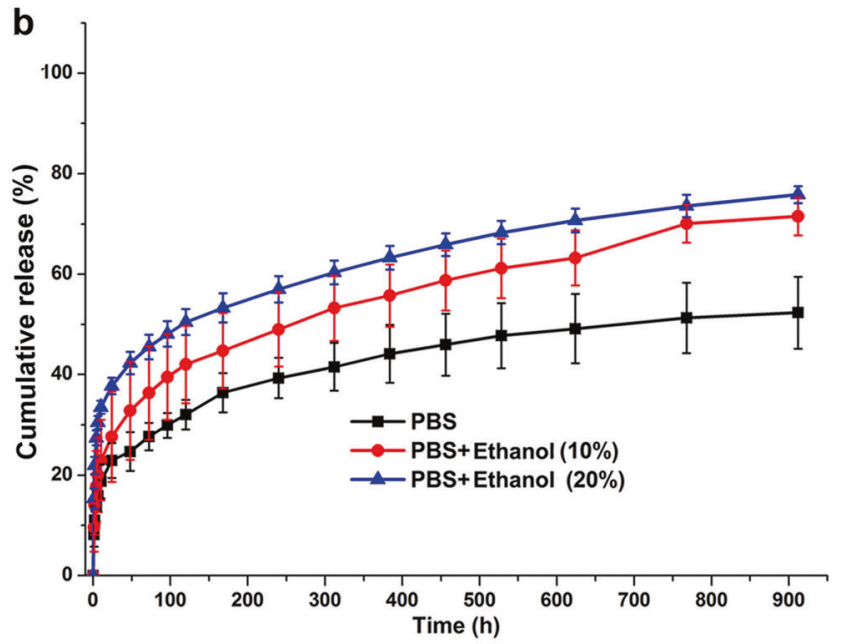

TP5 solution, which was approximately $90 \%$ in the first $6 \mathrm{~h}$. This indicated that PPSG could effectively reduce the burst release of TP5. In the following sustained release phase, the drug release was controlled and long-lasting. It could be concluded that the drug release of TP5-PPSG was much slower than that of the TP5 solution, and it was also positively related to the ethanol content of the release medium. Phospholipid is highly soluble in ethanol, so the rapid dissolution of phospholipid in a medium with a higher ethanol concentration was accompanied by a more rapid drug release. Moreover, the phospholipid dissolution in ethanol could be used to simulate the in vivo degradation of phospholipid, which was helpful in predicting the drug release behavior in the body.

The release kinetics of TP5 from PPSG were subsequently fitted to six classical mathematical models to explore the release mechanism and evaluated based on the correlation coefficient $\left(R^{2}\right)$ (Table 1). $R^{2}$ was $>0.9$ for the Higuchi model, the Ritger-Peppas model and the Weibull model. The results indicated that the drug release mechanism of PPSG could result from a combination of gel erosion, drug diffusion and dissolution, all of which contributed to the controlled and sustained drug release from PPSG.

In vivo pharmacokinetics study of TP5-PPSG

Because of its very short half-life in vivo (less than $30 \mathrm{~s}$ ) [28] and its low concentration after administration at a low dosage, TP5 is hard to quantify in a pharmacokinetics study. Therefore, fluorescently labeled TP5, 5-FAM-TP5, was chosen to study the pharmacokinetic behavior of TP5-loaded PPSG, and fluorescence spectrophotometry was used because it has high sensitivity for quantification. Drug concentration-time curves for 5-FAM-TP5 solution and PPSG formulations after subcutaneous injection in rats were drawn and compared. Fig. 5 shows that, in the 5-FAMTP5 solution group, the drug concentration in the plasma increased rapidly to approximately $14,000 \mathrm{ng} / \mathrm{mL}$ in the first half hour after injection and then decreased sharply, and the drug could not be detected at $24 \mathrm{~h}$. Conversely, the plasma drug concentration of the PPSG formulation increased slowly and peaked within $4 \mathrm{~h}$, followed by a gradual and stable decline from 4 to $720 \mathrm{~h}$. In addition, the drug concentration at $24 \mathrm{~h}$ was still almost as high as $200 \mathrm{ng} / \mathrm{mL}$, much higher than that in the solution group. Table 2 shows that both the half-life $\left(t_{1 / 2}\right)$ and the mean retention time (MRT) of 5-FAM-TP5-PPSG were significantly much longer than those of the free drug solution $(P<0.01)$, demonstrating the favorable sustained release characteristics of 


\begin{tabular}{|c|c|c|c|}
\hline \multirow[t]{2}{*}{ Zero-order: $y=a^{*} t+b$} & PBS & $y=0.04535 t+20.858$ & 0.79787 \\
\hline & $20 \%$ ethanol & $y=0.05665 t+35.292$ & 0.69285 \\
\hline First-order: $\ln (1-y)=a^{*} t+b$ & PBS & $\ln (1-y)=-0.0007 t+4.3702$ & 0.85871 \\
\hline \multirow[t]{3}{*}{ Higuchi: $y=a^{*} t^{0.5}+b$} & PBS & $y=1.4805 t^{0.5}+13.241$ & 0.9554 \\
\hline & $10 \%$ ethanol & $y=1.9915 t^{0.5}+16.024$ & 0.96959 \\
\hline & $20 \%$ ethanol & $y=1.8498 t^{0.5}+25.772$ & 0.9415 \\
\hline \multirow[t]{2}{*}{ Ritger-Peppas: $\ln y=a^{*} \ln t+b$} & PBS & $\ln y=0.25912 \ln t+2.2377$ & 0.99224 \\
\hline & $10 \%$ ethanol & $\ln y=0.26671 \ln t+2.4422$ & 0.99167 \\
\hline Weibull: $\ln \ln (1 /(1-y))=a^{*} \ln t+b$ & $20 \%$ ethanol & $\ln \ln (1 /(1-y))=0.27834 \ln t-1.6307$ & 0.98459 \\
\hline \multirow[t]{3}{*}{ Hixson-Crowell: $(1-y)^{1 / 3}=a^{*} t+b$} & PBS & $(1-y)^{1 / 3}=-0.00094 t+4.2921$ & 0.83928 \\
\hline & $10 \%$ ethanol & $(1-y)^{1 / 3}=-0.00148 t+4.2033$ & 0.90236 \\
\hline & $20 \%$ ethanol & $(1-y)^{1 / 3}=-0.00148 t+4.0156$ & 0.86937 \\
\hline
\end{tabular}

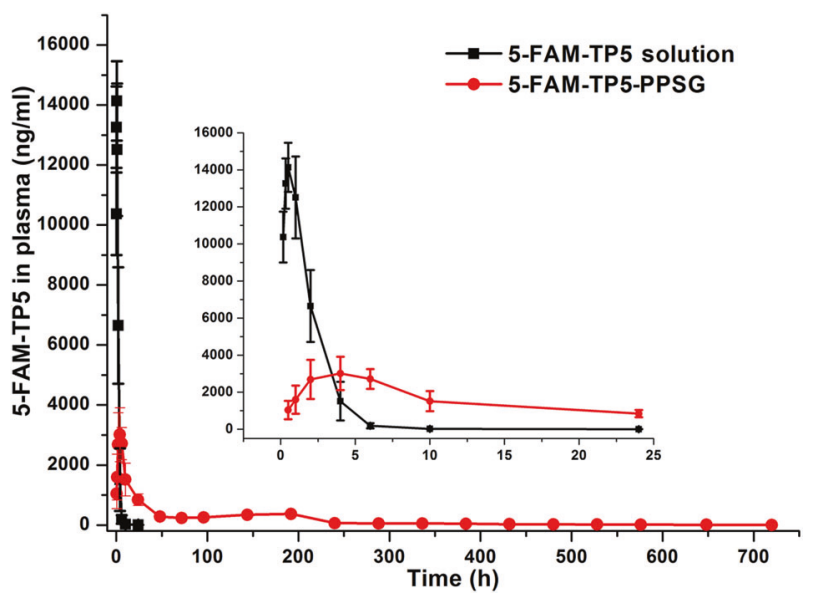

Fig. 5 Pharmacokinetic profiles of 5-FAM-TP5 solution and 5-FAMTP5-PPSG after subcutaneous injection at the dosage of $20 \mathrm{mg} / \mathrm{kg}$ body weight $(n=5)$

PPSG. Additionally, the area under the curve (AUC) for the PPSG group was significantly higher than that for the drug solution group, indicating the higher bioavailability of PPSG. The reason for the increased AUC could be the prolonged elimination phase $(24-720 \mathrm{~h})$, as well as the much higher 5-FAM-TP5 concentration during the elimination phase of the PPSG group than that of the solution group (5-720 h).

The above results clearly demonstrated that after loading into PPSG, 5-FAM-TP5 could be released in a controlled and sustained manner for as long as one month, which was largely due to the in situ-formed PPSG implant containing a high phospholipid concentration and having high viscosity as a drug release barrier.

In vivo pharmacodynamics assay

TP5 is an effective immunomodulatory agent that can improve an unbalanced immune system, including affecting T-cell function and improving SOD activity to a basal level $[7,18,29]$. Therefore,
Table 2. Pharmacokinetic parameters of 5-FAM-TP5 solution and 5-FAM-TP5-PPSG

\begin{tabular}{|c|c|c|c|}
\hline Parameters & 5-FAM-TP5 solution & 5-FAM-TP5-PPSG & $\begin{array}{l}\text { PPSG/ } \\
\text { solution }\end{array}$ \\
\hline$C \max (\mathrm{ng} / \mathrm{mL})$ & $14387.45 \pm 1316.72$ & $636.595 \pm 177.50$ & 0.04 \\
\hline$T \max (\mathrm{h})$ & $0.50 \pm 0.00$ & $4.00 \pm 0.00$ & 8.00 \\
\hline$t_{1 / 2(\mathrm{~h})}$ & $2.69 \pm 2.58$ & $114.00 \pm 45.24^{* *}$ & 42.32 \\
\hline $\begin{array}{l}\mathrm{AUCO} \rightarrow \mathrm{t}(\mathrm{ng} / \\
\left.\mathrm{mL} \mathrm{L}^{*} \mathrm{~h}\right)\end{array}$ & $29,793.19 \pm 4878.18$ & $36,780.13 \pm 2757.01 *$ & 1.23 \\
\hline $\begin{array}{l}\text { AUC0 } \rightarrow \infty \text { (ng/ } \\
\left.\mathrm{mL}^{*} \mathrm{~h}\right)\end{array}$ & $29,811.63 \pm 4876.72$ & $37,453.94 \pm 2638.67^{*}$ & 1.26 \\
\hline $\mathrm{MRTO} \rightarrow \mathrm{t}(\mathrm{h})$ & $1.503 \pm 0.16$ & $193.69 \pm 22.63^{* *}$ & 128.87 \\
\hline MRT0 $\rightarrow \infty(h)$ & $1.52 \pm 0.14$ & $206.95 \pm 22.99^{* *}$ & 135.88 \\
\hline
\end{tabular}

according to previous studies $[15,30,31]$, the T-SOD level and Tlymphocyte subset $\mathrm{CD} 4+/ \mathrm{CD} 8+$ ratios were determined in a pharmacodynamics study to evaluate the therapeutic effect of TP5-loaded PPSG on immunosuppressed rats.

$T$-SOD value measurement. The T-SOD activity of the plasma from rats after various treatments is shown in Fig. 6 . The T-SOD activity of the immunosuppression group was significantly lower than that of normal control rats $(P<0.001)$, indicating that the immunosuppressed animal model was established successfully and stably. After treatment with the TP5 formulations, the T-SOD activity of the immunosuppressed rats increased to the same level as normal rats $(P>0.05)$ and to significantly higher than that of the immunosuppressed group $(P<0.001)$, proving that both the TP5 solution and TP5-PPSG treatment could restore the plasma T-SOD activity of the immunosuppressed rats to normal levels. In addition, no significant difference was observed for the T-SOD 


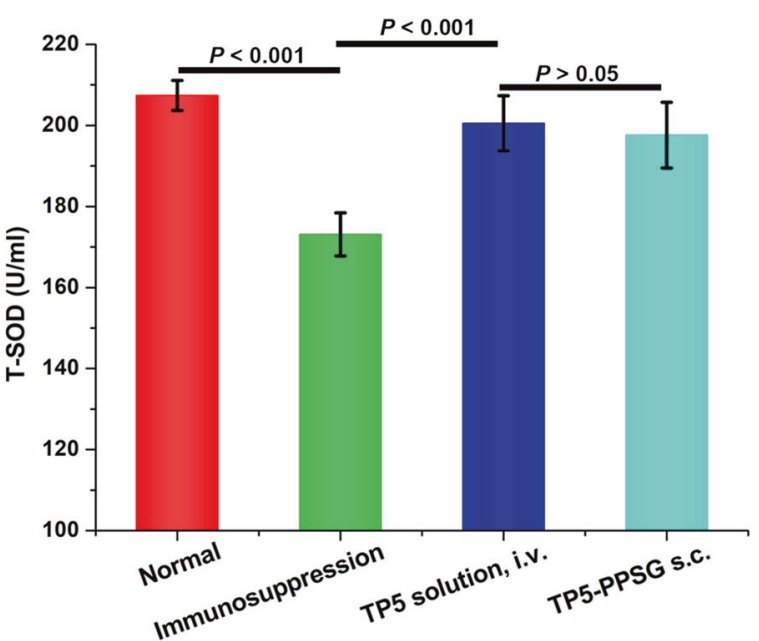

Fig. 6 The T-SOD activity of the plasma from rats of each group in the pharmacodynamics study $(n=5)$

\begin{tabular}{|c|c|c|c|}
\hline Group & $\begin{array}{l}\text { CD3+CD4+ } \\
(\%)\end{array}$ & $\begin{array}{l}\text { CD3+CD8+ } \\
\text { (\%) }\end{array}$ & $\begin{array}{l}\text { CD4+/CD8+ } \\
\text { (\%) }\end{array}$ \\
\hline Normal & $57.53 \pm 8.19$ & $28.53 \pm 4.15$ & $2.05 \pm 0.46$ \\
\hline Immunosuppression & $85.52 \pm 1.70$ & $14.06 \pm 1.62$ & $6.16 \pm 0.84^{* *}$ \\
\hline TP5 solution, s.c. & $55.43 \pm 9.10$ & $29.75 \pm 3.38$ & $1.88 \pm 0.36$ \\
\hline TP5-PPSG, s.c. & $56.70 \pm 3.85$ & $28.73 \pm 3.72$ & $2.01 \pm 0.40^{\# ; ; ; \&}$ \\
\hline \multicolumn{4}{|c|}{$\begin{array}{l}\text { Data presented as mean } \pm \text { SD }(n=5) \\
{ }^{* *} P<0.01 \text { versus normal } \\
\# P>0.05 \text { versus normal } \\
\$ P>0.05 \text { versusTP5 solution } \\
{ }^{\&} P<0.01 \text { versus immunosuppression }\end{array}$} \\
\hline
\end{tabular}

values of the TP5 solution group and the TP5-PPSG group $(P>$ 0.05).

SOD catalyzes the transformation of the superoxide free radical $\left(\mathrm{O}^{2-}\right)$ into hydrogen peroxide $\left(\mathrm{H}_{2} \mathrm{O}_{2}\right)$ and elemental oxygen $\left(\mathrm{O}^{2}\right)$ by a disproportionation process, which effectively alleviates the toxicity of superoxide free radicals [30]. Therefore, SOD is an important antioxidase and is closely associated with digestive diseases, kidney disease, cardiovascular disease, inflammation, immunity, tumors, and blood disease [31]. Previous studies showed that the T-SOD concentration in healthy rats was approximately $200 \mathrm{U} / \mathrm{mL}$, but it deceased when the rats were immunosuppressed $[15,32]$, indicating that the T-SOD level could indirectly reflect the immunity level. In this study, the T-SOD level of rats treated with single dose of TP5-PPSG $(15 \mathrm{mg} / \mathrm{kg})$ was similar to that of rats administered with a TP5 solution daily $(0.6$ $\mathrm{mg} / \mathrm{kg}$ per day) in a 14-day test, implying that TP5-PPSG could greatly reduce the dosing frequency without affecting the immunoregulatory efficacy.

Analysis of lymphocyte subsets by flow cytometry. Table 3 shows that the ratio of CD4+/CD8 + T cells in immunosuppressed rats was significantly higher than that in normal rats $(6.16 \pm 0.84$ versus $2.05 \pm 0.46, P<0.01)$, demonstrating the successful establishment of an immunosuppression model. Furthermore, consistent with the results of the T-SOD assay, no significant difference was seen between the $C D 4+/ C D 8+$ ratios of normal rats and those in the TP5-PPSG group $(P>0.05)$, which demonstrated that TP5-loaded
PPSG could regulate dysfunctional T-lymphocytes to normal. Even more exciting was that no significant difference was seen between the results for the daily injected TP5 solution group and the single administered TP5-PPSG group $(P>0.05)$, which demonstrated an equivalent immune-modulating effect of these different treatments for immunosuppression.

T-lymphocytes are divided into various subsets based on their phenotype and function, and the different subsets express different surface markers. CD3 is a common surface marker expressed by all T-lymphocytes, while CD4 and CD8 are expressed on the surface of inducer-helper $\mathrm{T}$ cells $(\mathrm{Ti} / \mathrm{Th})$ and suppressorcytotoxic T cells (Ts/Tc), respectively. CD4+ and CD8+ indicate mature T-lymphocytes [30], and T-lymphocytes of normal humans have been shown to have a stable CD4+/CD8 + ratio (1.5-2.0) [13], which changes if an immune abnormality is present. Therefore, the $C D 4+/ C D 8+$ ratio could reflect the immune status of an animal. However, cross expression of surface markers exists in lymphocyte subtypes; for example, CD4 is expressed in monocytes, and CD8 is expressed in NK cells, whereas CD3 is not expressed on these two cell types. Therefore, we used CD3 labeling to distinguish various types of T-lymphocytes.

Both the T-SOD test and the T-lymphocyte CD4+/CD8+ ratio assay in this study demonstrated that a single dose of TP5-PPSG (15 mg/kg, s.c.) had the same immune-modulating capacity as that of a daily administered TP5 solution $(0.6 \mathrm{mg} / \mathrm{kg}$ per day, s.c.), effectively restoring the immunosuppressed rats to normal. The results also demonstrated the sustained and long-lasting pharmacokinetic behavior of TP5-PPSG, which would benefit the clinical use of TP5 by reducing the administration frequency and greatly improving patient compliance. In addition, we previously demonstrated that the inflammation response in rabbits caused by PPSG after a subcutaneous injection was low and acceptable, indicating the biocompatibility of the TP5 drug delivery system [20].

In conclusion, we successfully developed a TP5 delivery system with a sustained drug release profile and long-acting therapeutic effect in this study. TP5-PPSG could be prepared by a simple onestep stirring method, and the changes in its physicochemical properties during a phase transition contributed to a sustained drug release of as long as a month both in vitro and in vivo. As a result, compared with a daily injected TP5 solution, a single dose of TP5-PPSG showed a comparable effect of restoring the T-SOD level and the CD4+/CD8+ ratio to normal in immunosuppressed rats, indicated a long-lasting immunoregulatory effect of TP5PPSG. Therefore, patient compliance could be greatly improved by reducing the administration frequency. In summary, TP5-PPSG has great potential for the sustained delivery of TP5 in the clinic because of its simple manufacturing process, good biocompatibility and long-lasting immunoregulatory efficacy.

\section{ACKNOWLEDGEMENTS}

This work was supported by grants from the National Natural Science Foundation of China (81690261 and 81673359).

\section{AUTHOR CONTRIBUTION}

T.G., T.Z. and Z.-r.Z. conceived the ideas and designed the experiments; T.Z., X.Q., X.C. and W.-h.L. conducted the experiments; T.Z. and T.G. interpreted the data and wrote the manuscript. All authors agree to be accountable for all aspects of the work.

\section{ADDITIONAL INFORMATION}

Competing interests: The authors declare no competing interests.

\section{REFERENCES}

1. Goldstein G, Audhya TK. Thymopoietin to thymopentin: experimental studies. Surv Immunol Res. 1985;4(Suppl 1):1-10. 
2. Colle R, Ceschia T, Colatutto A, Biffoni F. Use of thymopentin in autoimmune hemolytic anemia due to chronic lymphocytic leukemia. Curr Ther Res. 1988;44:1045-9.

3. Sundal $E$, Bertelletti D. Thymopentin treatment of rheumatoid arthritis. ArzneimForsch. 1994;44:1145-9.

4. Bodey B, Bodey B, Siegel SE, Kaiser HE. Review of thymic hormones in cancer diagnosis and treatment. Int J Immunopharmacol. 2000;22:261-73.

5. Coppola S, Buccoliero G, Laddago V, Monno L, Perrone A, Guida G, et al. Topical thymopentin therapy in HIV positive patients with recurrent oral candidiasis: a pilot study. New Microbiol. 1996;19:351-5.

6. Xiaojing C, Yanfang L, Yanqing G, Fangfang C. Thymopentin improves cardiac function in older patients with chronic heart failure. Anatol J Cardiol. 2017;17:24-30.

7. Singh VK, Biswas S, Mathur KB, Haq W, Garg SK, Agarwal SS. Thymopentin and splenopentin as immunomodulators. Curr Status Immunol Res. 1998;17:345-68.

8. Kondratyev MS, Lunin SM, Kabanov AV, Samchenko AA, Komarov VM, Fesenko $\mathrm{EE}$, et al. Structural and dynamic properties of thymopoietin mimetics. J Biomol Struct Dyn. 2014;32:1793-801.

9. Gonser S, Weber E, Folkers G. Peptides and polypeptides as modulators of the immune response: thymopentin--an example with unknown mode of action. Pharm Acta Helv. 1999;73:265-73.

10. Audhya TK, Goldstein G. Thymopentin: stability considerations and potency by various routes of administration. Surv Immunol Res. 1985;4(Suppl 1):17-23.

11. Li YZ, Sun X, Gong T, Liu J, Zuo J, Zhang ZR. Inhalable microparticles as carriers for pulmonary delivery of thymopentin-loaded solid lipid nanoparticles. Pharm Res. 2010;27:1977-86.

12. Audhya T, Goldstein G. Thymopentin (TP-5) potency in vivo is enhanced by slow infusion. Int J Pept Protein Res. 1983;22:568-72.

13. Yin $Y$, Chen D, Qiao M, Lu Z, Hu H. Preparation and evaluation of lectinconjugated PLGA nanoparticles for oral delivery of thymopentin. J Control Release. 2006;116:337-45.

14. Yin $Y$, Chen D, Qiao M, Wei X, Hu H. Lectin-conjugated PLGA nanoparticles loaded with thymopentin: ex vivo bioadhesion and in vivo biodistribution. J Control Release. 2007;123:27-38.

15. Wu C, Zhang M, Zhang Z, Wan KW, Ahmed W, Phoenix DA, et al. Thymopentin nanoparticles engineered with high loading efficiency, improved pharmacokinetic properties, and enhanced immunostimulating effect using soybean phospholipid and PHBHHx polymer. Mol Pharm. 2014;11:3371-7.

16. Wei G, Jin L, Xu L, Liu Y, Lu W. Preparation, characterization and in vivo pharmacodynamic evaluation of thymopentin loaded poly(lactide acid)/poly(lactideco-glycolide acid) implants. Int J Pharm. 2010;398:123-9.

17. Zhang Y, Wu X, Han Y, Mo F, Duan Y, Li S. Novel thymopentin release systems prepared from bioresorbable PLA-PEG-PLA hydrogels. nt J Pharm. 2010; 386:15-22.

18. Lin S, Cai B, Quan G, Peng T, Yao G, Zhu C, et al. Novel strategy for immunomodulation: dissolving microneedle array encapsulating thymopentin fabricated by modified two-step molding technology. Eur J Pharm Biopharm. 2018; 122:104-12.

19. Zhong Y, Chen L, Zhang Y, Li W, Sun X, Gong T, et al. Vesicular phospholipid gels using low concentrations of phospholipids for the sustained release of thymopentin: pharmacokinetics and pharmacodynamics. Pharmazie. 2013;68:811-5.

20. Zhang T, Peng Q, San FY, Luo JW, Wang MX, Wu WQ, et al. A high-efficiency, lowtoxicity, phospholipids-based phase separation gel for long-term delivery of peptides. Biomaterials. 2015;45:1-9.

21. Simmons BA, Taylor CE, Landis FA, John VT, McPherson GL, Schwartz DK, et al. Microstructure determination of AOT+ phenol organogels utilizing small-angle X-ray scattering and atomic force microscopy. J Am Chem Soc. 2001; 123:2414-21.

22. Ki MH, Lim JL, Ko JY, Park SH, Kim JE, Cho HJ, et al. A new injectable liquid crystal system for one month delivery of leuprolide. J Control Release. 2014;185:62-70.

23. Sauerbrey A, McPherson JP, Zhao SC, Banerjee D, Bertino JR. Expression of a novel double-mutant dihydrofolate reductase-cytidine deaminase fusion gene confers resistance to both methotrexate and cytosine arabinoside. Hum Gene Ther. 1999;10:2495-504.

24. Ravar F, Saadat E, Gholami M, Dehghankelishadi P, Mahdavi M, Azami S, et al. Hyaluronic acid-coated liposomes for targeted delivery of paclitaxel, in-vitro characterization and in-vivo evaluation. J Control Release. 2016;229:10-22.

25. Peng Q, Zhang ZR, Gong T, Chen GQ, Sun X. A rapid-acting, long-acting insulin formulation based on a phospholipid complex loaded PHBHHx nanoparticles. Biomaterials. 2012;33:1583-8.

26. Wang M, Shan F, Zou Y, Sun X, Zhang ZR, Fu Y, et al. Pharmacokinetic and pharmacodynamic study of a phospholipid-based phase separation gel for once a month administration of octreotide. J J Control Release. 2016;230:45-56.

27. Gurfinkel J, Aserin A, Garti N. Interactions of surfactants in nonionic/anionic reverse hexagonal mesophases and solubilization of a-chymotrypsinogen $\mathrm{A}$. Colloids Surf A. 2011;392:322-8.

28. Tischio JP, Patrick JE, Weintraub HS, Chasin M, Goldstein G. Short in vitro half-life of thymopoietin32--36 pentapeptide in human plasma. Int J Pept Protein Res. 1979;14:479-84.

29. Zhu MX, Wan WL, Li HS, Wang J, Chen GA, Ke XY. Thymopentin enhances the generation of T-cell lineage derived from human embryonic stem cells in vitro. Exp Cell Res. 2015;331:387-98.

30. Wang J, Lu WL, Liang GW, Wu KC, Zhang CG, Zhang $X$, et al. Pharmacokinetics, toxicity of nasal cilia and immunomodulating effects in Sprague-Dawley rats following intranasal delivery of thymopentin with or without absorption enhancers. Peptides. 2006;27:826-35.

31. Tang X, Yin C, Zhang F, Fu Y, Chen W, Chen Y, et al. Measurement of subgroups of peripheral blood $\mathrm{T}$ lymphocytes in patients with severe acute respiratory syndrome and its clinical significance. Chin Med J. 2003;116:827-30.

32. Treitinger A, Spada C, Verdi JC, Miranda AF, Oliveira OV, Silveira MV, et al. Decreased antioxidant defence in individuals infected by the human immunodeficiency virus. Eur J Clin Invest. 2000;30:454-9. 